\title{
Capsule colique ou coloscopie virtuelle : le match est-il fini ?
}

\section{Colon Capsule or CT Colonography: Is Match Over?}

\section{Heresbach}

(C) Lavoisier SAS 2015

Cinq à 10\% des coloscopies sont incomplètes et nécessitent une exploration complémentaire, le plus souvent par la coloscopie virtuelle (CTC). La performance de la vidéocapsule colique de seconde génération (VCC) invite toutefois à reconsidérer cette stratégie.

La présente étude [1], même si elle s'adresse au sousgroupe de patients avec coloscopie incomplète, a le mérite de comparer chez les mêmes patients les deux techniques et répond à la question posée en pratique quotidienne. Cent patients, inclus en deux ans, ont été explorés par VCC et CTC et une seconde coloscopie était réalisée si un polype $\geq 6 \mathrm{~mm}$ était détecté par l'une des deux méthodes. L'exploration colique par VCC était complète dans $98 \%$ des cas. La préparation colique était jugée bonne pour la VCC et la CTC dans respectivement $83 \%$ (IC $95 \% ; 74-90$ ) et $90 \%$ (IC $95 \% ; 82-95)$. Un polype $\geq 6 \mathrm{~mm}$ a été détecté par VCC ou CTC ou les deux chez 25 patients, et la coloscopie a confirmé ce résultat dans $92 \%$ des cas $(24 / 25)$ avec un faux-positif en VCC et deux en CTC. La VCC et CTC détectaient respectivement 24 et 12 des 24 patients avec un polype $\geq 6 \mathrm{~mm}$ avec une VPP de $96 \%$ (IC:95\%; 78-99) et $86 \%$ (IC:95\%; 56-97). Le taux de détection des polypes $\geq 10 \mathrm{~mm}$ par la VCC ou la CTC étaient respectivement de $100 \%$ et $50 \%$ et les VPP $83 \%$ et $100 \%$. Aucun effet secondaire majeur n'a été attribué à l'un ou l'autre mode d'exploration mais 28 ont présenté des effets indésirables mineurs liés à la préparation colique. Parmi les 74 cas sans lésion à la VCC ou en CTC, aucun cancer colorectal «manqué » n'a été diagnostiqué chez ces patients après un suivi moyen de 20 mois par téléphone !

Au vu de ces résultats, comme on dit, le match est plié ! Est-il besoin de jouer les prolongations pour obtenir une spécificité comparée ? Possiblement, car c'est la valeur prédictive négative qui importe pour définir la stratégie adaptée (surveillance? nouvelle coloscopie ? chirurgie ?)

On doit donc réaliser cette fameuse étude comparant VCC et CTC et coloscopie totale.

\section{Référence}

1. Spada C, Hassan C, Barbaro B, et al. Colon capsule versus CT colonography in patients with incomplete colonoscopy: a prospective, comparative trial. Gut 2015;64:272-81.

D. Heresbach $(\bowtie)$

Unité d'endoscopie et de consultation digestive, centre hospitalier de Redon, 8, rue Étienne Gascon, F-35603 Redon

e-mail : denis.heresbach@ch-redon.fr 